\title{
Relationship between Material Pitting AND Cavitation Field Impulsive Pressures
}

\author{
Jin-Keun Choi $^{1}$ and Georges L. Chahine \\ DYNAFLOW, INC., 10621-J Iron Bridge Road, Jessup, MD, USA \\ $\underline{\text { www.dynaflow-inc.com }}$
}

\begin{abstract}
1. Abstract
Material pitting from cavitation has been used on and off as an indicator of the vague concept of 'cavitation intensity'. Periodically, some researchers suggest the use of pitting tests as a means to provide quantitative measurements of the amplitude of the impulsive pressures in the cavitation field, especially when combined with Tabor's formula or with finite element computations with idealized synthetic loads. This paper examines the viability of such a suggested method using fully coupled bubble dynamics and material response, and strongly concludes that the method provides at best a qualitative assessment of the cavitation erosion potential. Peak pressures deduced from pit geometry are significantly lower than the ones actually applied. In addition the correspondence is highly dependent on the way the load is applied and different loading scenarios with the same amplitude of the cavitation impulsive pressure result in different pit aspect ratios.
\end{abstract}

Keywords: cavitation erosion; erosion modeling; cavitation pit; bubble dynamics; fluid structure interaction

\section{Introduction}

A material surface, which is exposed to cavitation, progressively erodes over time due to local high amplitude short duration and small footprint loads from repeated individual and collective cavitation bubble collapse [1]. Proper modeling of the physical phenomena at play is complex and requires understanding and description of both the two-phase fluid flow and the material dynamics as well as their interaction. Cavitation initiates from bubble nuclei in the liquid, which when exposed to low pressures grow explosively then collapse violently when the pressure recovers, thus generating very high local pressures and shock waves [1-4]. When a bubble collapses onto a material surface, a reentrant micro jet forms in the largely deformed bubble, vectors towards the material and impacts its surface with shock waves forming in the subsequent dynamics [5-9]. The flow due to the bubble collapse and the reentrant jet impact generate high impulsive stress into the material. When these exceeds the elastic limit of the material, permanent deformation occurs and a microscopic pit is generated [10]. This initial phase of material response to the cavitation field, the "incubation period", does not involve any mass loss. With repeated impacts, hardening of the material surface layer develops, which could be used for material peening [11,12], then pits accumulate and finally micro-failures occur resulting in material removal and weight loss.

I'Corresponding author (jkchoi@dynaflow-inc.com) 
More recently, there have been renewed effort to correlate the cavitation field impulsive pressure and the measured pits characteristics [7,27,28], in which attempts have been made to correlate the experimentally measured ratios of pit depth to diameter to the pressure filed using the spherical indentation relationships developed by Tabor [29] and making, without real justification, the major assumption that spherical indentations (rigid load/material interface and strain rates, $\sim 0.05 \mathrm{~s}^{-1}$ ) and collapsing bubbles (deforming liquid/material interface and strain rates, $\sim 10^{4} \mathrm{~s}^{-1}$ ) produce similar loads on the material. An effort to include the strain rate dependency of the stress-strain curves was attempted in [28] but static loads only were still considered. Recently, finite element method analysis was also conducted [30-31] using the same basis for the load as in the Tabor approach, i.e. time independent idealized constant pressure load, and obviously, due to the over-simplified parameters, an almost one-to-one relationship between the loads and the pit geometry characteristics was found. The time dependent analysis in [32] confirmed that such one-to-one relation is achieved only when the time scale of the load is larger than $10^{5} \mu \mathrm{s}$ (see Fig.5 of [32]), which is huge compared to the duration of bubble dynamics generated peak pressures.

The believers in the pitting technique claim that the method allows the material to be used as a high fidelity recorder of the cavitating field pressures and that the results would be independent of the material used [16, 27, 31]. Actually, a first strong limitation of this statement is that only pressures that lead to stresses higher than the elastic limit of each particular material will form pits and all lower pressures cannot be measured. Thus, each material acts as a high pass filter and records only the pressures above a cutoff value, which is material dependent. Other even more significant limitations are examined in more details in this paper using advanced bubble dynamics-material dynamics interaction modeling techniques [7, 8, 33] and 
stem from the examination of the quantitative relationship between pit characteristics and actual dynamic pressure loads.

In order to investigate this relationship, a numerical fluid-material interaction approach is undertaken to investigate pitting formation from the combined bubble dynamics and material mechanics viewpoints. Fully coupled Fluid Structure Interaction (FSI) simulations are conducted and provide both the actual liquid generated pressures and the resulting material pit characteristics. Using these results, the actual pressure applied on the material surface is compared with the one deduced from the pit geometry using the Tabor equations [29]. The same approach is also applied using synthetic loads (prescribed loading, no FSI) as published in previous work [30-33] where the applied load is specified and the resulting material pits deduced and the results compared to the Tabor predictions.

\section{Material / Fluid Interaction Simulations}

In this study, correlation between the cavitation pressures in the liquid and the resulting pit characteristics is studied using FSI coupled material finite element method simulations with fluid dynamic simulations of bubble collapse of various intensities. We describe briefly below the methods used and refer the reader to much detailed descriptions in references [7-9] as well as other references cited below.

\subsection{Bubble Dynamics}

The numerical approach applied to model material pitting is part of a general hybrid FSI approach we developed to simulate fluid structure interaction problems involving shock and bubble dynamics encountered in cavitation and underwater explosion bubbles [32-35]. During a major portion of the bubble growth and collapse history, the velocities in the liquid are much smaller than the liquid speed of sound, and an incompressible approach is justified. A potential flow boundary element code, $3 \operatorname{DYNAFS} \mathrm{BEM}^{\odot}[32,33,36]$ is used during this time period. On the other hand, shock waves and strong compressibility of the liquid come into play during the last stage of the bubble collapse and following reentrant jet impact on the material. A time decomposition approach is then used to switch for the incompressible solution to fully compressible solvers such as GEMINI [36] or 3DYNAFS-COMP ${ }^{\circledR}$ [39]. This time decomposition hybrid procedure combining incompressible and compressible solvers to capture the full dynamics has been described in details in [37]. The procedure takes advantage of the capabilities of 3DYNAFS-BEM ${ }^{\odot}$, to produce very accurate capturing of the reentrant jet [40], and GEMINI and 3DYNAFS-COMP ${ }^{\odot}$, which have been proven very good at capturing shock dynamics and resulting pressures on the boundaries [41]. Description of both methods and the coupling procedure can be found in $[35,39]$. Illustrations of the results are shown later below.

\subsection{Material Response}

The dynamics of the material is modeled by the finite element model DYNA3D, which is a non-linear explicit structure dynamics code developed by the Laurence Livermore National Laboratory [42]. The US Navy version used in this paper is named DYNAN. The structural code computes the material deformation with the loading being provided here by the fluid solution (either 3DYNAFS-BEM ${ }^{\circledR}$, 3DYNAFS-COMP ${ }^{\circledR}$, or GEMINI). DYNAN uses a lumped mass 
formulation for efficiency. This produces a diagonal mass matrix $\mathbf{M}$, to express the dynamics equation as:

$$
\mathbf{M} \frac{d^{2} \mathbf{x}}{d t^{2}}=\mathbf{F}_{\mathrm{ext}}-\mathbf{F}_{\mathrm{int}},
$$

where $\mathbf{F}_{\text {ext }}$ represents the applied external forces, and $\mathbf{F}_{\text {int }}$ the internal forces. The acceleration, $d \mathbf{x}^{2} / d t^{2}$, for each element is obtained through an explicit temporal central difference method. Additional details on the general formulation can be found in [42].

\subsection{Cavitation and Material Interactions}

Material-fluid interaction effects are captured in the simulations by coupling at each computation time step the fluid codes and DYNA3D using a coupler interface. The step-by-step coupling is achieved by following the numerical procedure below:

Step 1: The relevant fluid code computes the pressures at all material surface nodes.

Step 2: In response to this pressure loading, the material code computes material deformations, stresses, strains, and the surface node velocities.

Step 3: The coordinates and the velocities of the material surface nodes then define new boundary conditions for the fluid code at the following time step.

Step 4: The relevant fluid code then solves the flow field using the material surface node positions and normal velocities and deduces the liquid pressures at the material surface for the next time step, looping back to Step 2.

This procedure is illustrated in Figure 1. Additional details on the methods described above can be found in [37].

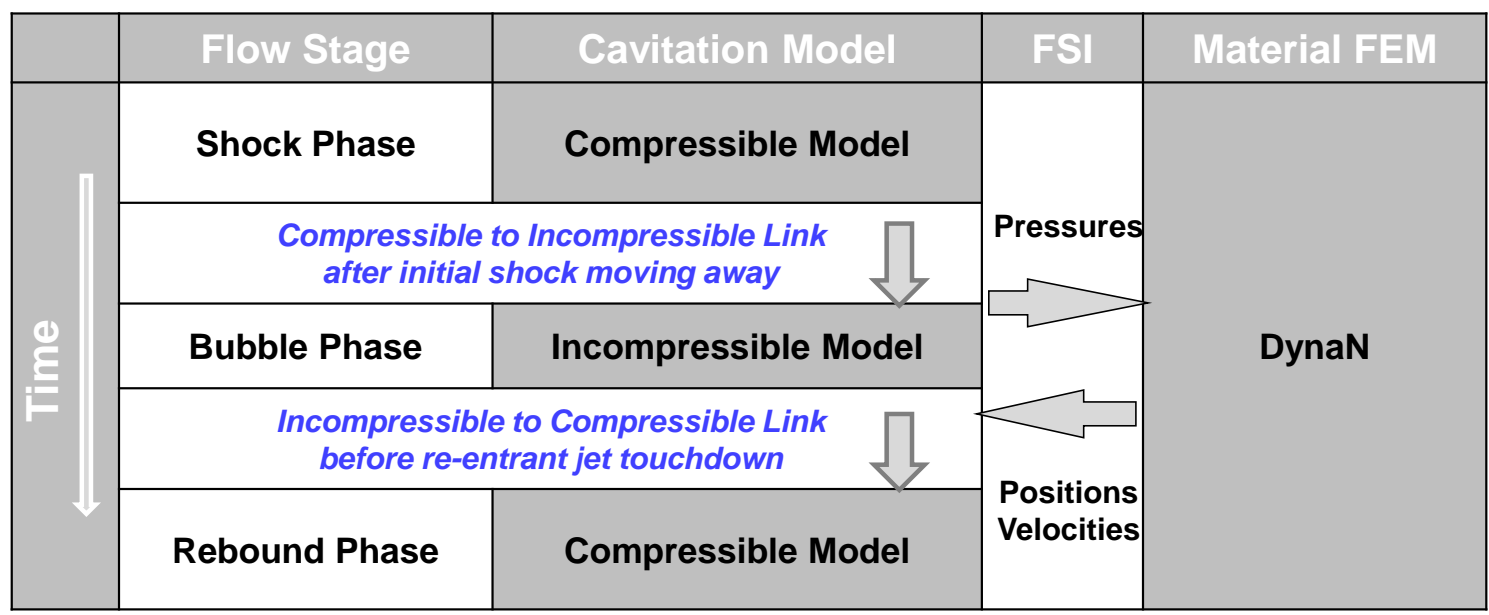

Figure 1. Schematic diagram of the numerical approach used to simulate the interaction between a cavitation bubble and a material surface.

\subsection{Synthetic Loading}

Another procedure to examine the validity of the Tabor equation approach is for us to use the finite element method described above and provide input excitation synthetically using an analytical representation, which corresponds to measured typical cavitation events pressure 
signals $[13,41]$. Compared to fully coupled simulations, where it is rather lengthy to vary the impact pressure magnitude and duration systematically, synthetic loading enables one to study more easily the effects of systematic variations of the magnitude, duration, and spatial extent of the impact loads. Previous numerical and experimental studies indicate that the pressure peaks in the cavitation fields can be represented well with a Gaussian function varying both in space and time for both ultrasonic and hydrodynamic cavitation conditions [13]. This representation (usually with the simplification of ignoring the time dependency) has been also adopted by other researchers in the field $[13,30,31]$.

Thus, in this paper, we consider simulations where, in addition to the actual computed bubble collapse pressures, idealized time and space varying impact pressure loadings, $P(r, t)$, in the following form are imposed:

$$
P(r, t)=P_{0} e^{-\left(\frac{t}{\Delta t}\right)^{2}} e^{-\left(\frac{r}{\Delta r}\right)^{2}},
$$

where $P_{0}$ is the amplitude of the pressure pulse, $\Delta t$ is its characteristic duration, and $\Delta r$ is its footprint characteristic radius.

\subsection{Materials Considered}

In this study of the correlation between liquid pressures loading the material and pit characteristics, four metals (Aluminum 1100, Aluminum 7075, Nickel Aluminum Bronze, and Stainless Steel A2205) are considered. These metals were modeled using elastic-plastic models with linear slopes (constant moduli), one for the initial elastic regime and the second, a tangent modulus, for the plastic regime as shown in Figure 2. The corresponding material parameters are shown in Table 1. A more complete model, which includes strain rate effects, the Johnson-Cook model [44], has also been used but the results are not shown here to simplify the presentation and illustrate the issues with using pitting results to deduce the loads.

\begin{tabular}{cccccc}
\hline $\begin{array}{c}\text { Metallic } \\
\text { Material }\end{array}$ & $\begin{array}{c}\text { Yield Stress } \\
(\mathbf{M P a})\end{array}$ & $\begin{array}{c}\text { Young's } \\
\text { Modulus } \\
(\mathbf{G P a})\end{array}$ & $\begin{array}{c}\text { Tangent } \\
\text { Modulus } \\
(\mathbf{M P a})\end{array}$ & $\begin{array}{c}\text { Elongation } \\
\text { at Break }\end{array}$ & $\begin{array}{c}\text { Density } \\
\left(\mathbf{g} / \mathbf{c m}^{\mathbf{3}}\right)\end{array}$ \\
\hline Al 1100 & 165 & 69 & 1,449 & 0.03 & 2.71 \\
NAB & 360 & 110 & 1,216 & 0.25 & 7.64 \\
Al 7075 & 503 & 71.7 & 670 & 0.11 & 2.81 \\
SS A2205 & 515 & 190 & 705 & 0.35 & 7.88 \\
\hline
\end{tabular}

Table 1. Properties of the material investigated in this study. 


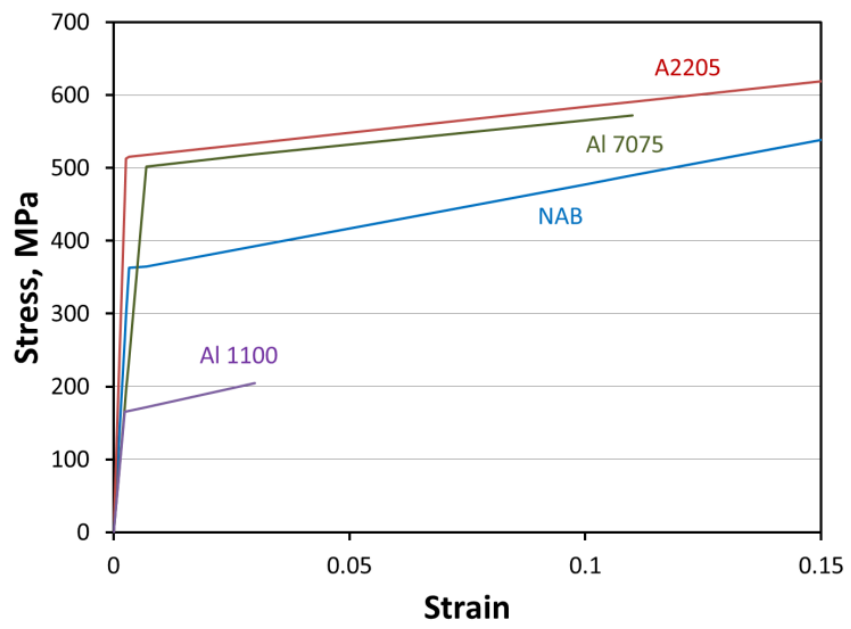

Figure 2. Simple assumed elastic-plastic stress-strain relations of the four materials investigated in this study.

\section{Deducing loads from Tabor Equation}

In Tabor's approach [29], a spherical indentation characterized by its diameter $D$ and depth $h$ as illustrated in Figure 3 is considered. The mean strain, $\varepsilon_{m}$, resulting from forming the pit in the material is estimated by:

$$
\varepsilon_{m}=k \frac{D}{2 R},
$$

where $R$ is the radius of the sphere that fits the spherical indent and $k$ is a coefficient close to 0.2 [28]. From geometrical relations of the spherical shape, Equation (3) can be re-written as:

$$
\varepsilon_{m}=0.8 \frac{h / D}{1+4(h / D)^{2}}=0.8 \frac{\xi}{1+4 \xi^{2}} \quad \text { with } \quad \xi=h / D .
$$

This equation shows that the mean strain associated with a spherical pit is related to the geometric aspect ratio, $\xi$, which is usually measured experimentally to represent the material deformation factor from pitting data and deduce the corresponding load [27, 28].

The corresponding maximum strain is then deduced from this mean strain assuming an exponential strain distribution of exponent, $\theta$, in the depth direction [27]:

$$
\varepsilon_{\max }=\varepsilon_{m}(1+\theta) .
$$

The users of this method report that $\theta$ can be obtained experimentally and give values for the materials used in this study [7,27].

From the maximum strain, the corresponding maximum stress can be deduced using the stress-strain relations of the material. In this approach, the maximum stress is assumed to occur at the surface of the material, and for the proponents of using pitting to measure liquid pressures, this stress is equated to the peak of the impulsive cavitation field pressure at the material surface. 


\section{Material Loading}

\subsection{Pressure Driving Bubble Collapse}

It is known that microbubbles or nuclei are omnipresent in liquids and, once excited by local pressures decreasing below a critical value (a commonly used value is the liquid vapor pressure), they grow explosively until their internal pressure drops below the liquid local pressure $[3,4,45]$. The bubbles then collapse violently, producing intense pressures, emitting sound, and eroding any solid surfaces in their proximity. To simulate a bubble dynamics pressure field under such a condition, we consider a nucleus initially at equilibrium with the surrounding liquid and we subject it to a time dependent pressure field, which would correspond to what the bubble would see while moving in the flow close to the material surface. We impose first a pressure drop to a value below the bubble critical pressure, keep this pressure for a prescribed time, $\Delta t$, then increase it to a high value, which is kept until the end of the computation.

Figure 4 illustrates this for the case of a bubble of initial radius $R_{0}=50 \mu \mathrm{m}$ at equilibrium in the liquid at $1 \mathrm{~atm}\left(10^{5} \mathrm{~Pa}\right)$. The pressure is then suddenly decreased to $10^{3} \mathrm{~Pa}$, and kept there for $\Delta t=2.415 \mathrm{~ms}$, and then is increased sharply to $P_{d}=10 \mathrm{MPa}$. Figure 4 also shows the response of a spherical bubble to this pressure function (in reality, the bubble will strongly deform as we will see below but this illustrates roughly the bubble volume change) obtained by integrating the Rayleigh-Plesset equation. It is seen that, as soon as the ambient pressure drops to the low value $\left(10^{3} \mathrm{~Pa}\right)$, the bubble responds and starts growing. The bubble cannot reach an equilibrium size and continues to grow during $\Delta t$ until the sudden pressure increase to $P_{d}$ occurs. A maximum bubble size, $R_{\max }$, is reached shortly after the overpressure is imposed due to liquid inertia. This is followed by a strong collapse of the bubble.

In this study, fully coupled FSI simulations were conducted with the magnitude of the collapse driving pressure, $P_{d}$, varying between $0.1 \mathrm{MPa}$ and $20 \mathrm{MPa}$. The duration of the pressure drop was varied such that the achieved bubble maximum radius, $R_{\max }$, achieved the sizes $0.5,1.0,1.5$ and $2.0 \mathrm{~mm}$. The bubble standoff, $X$, from the nearby material surface was also varied to correspond to $0.5,0.75,1.0$, and $1.5 R_{\max }$. 


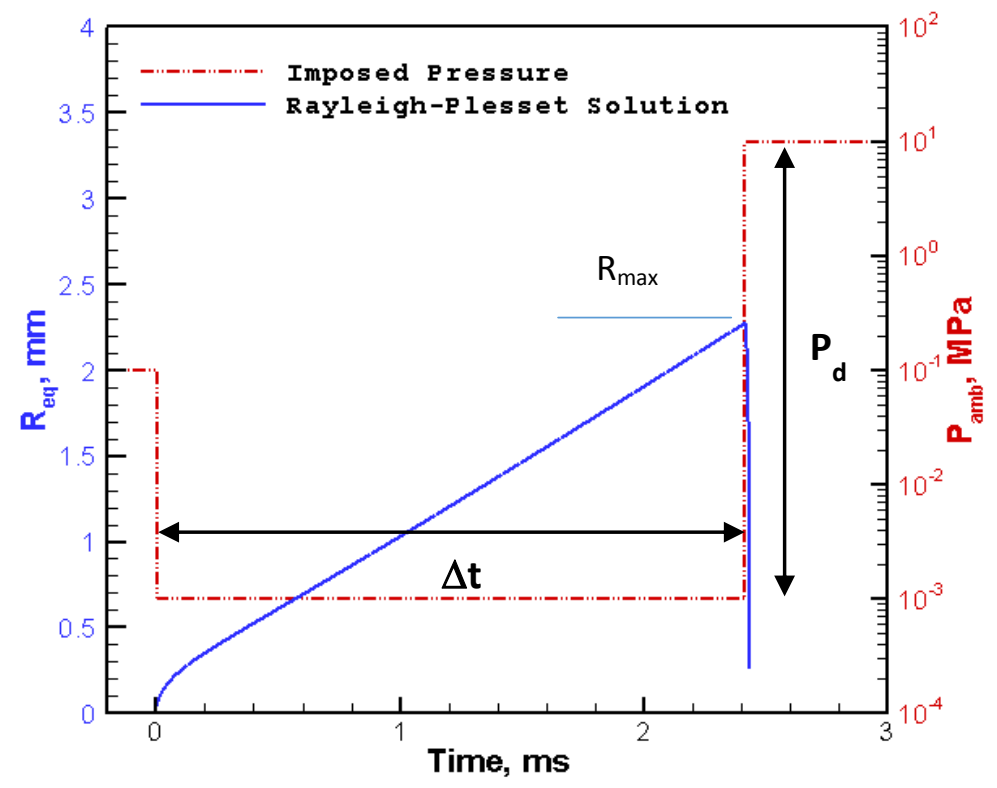

Figure 4. Imposed time dependent pressure field (red curve) around a spherical bubble of 50 um initial radius and resulting bubble radius vs. time (blue curve) as predicted by the solution of the Rayleigh-Plesset equation.

\subsection{Numerical Discretization}

The FSI simulations were conducted during the bubble collapse phase when the stresses caused material response or deformation. For the liquid bubble dynamics simulations an axisymmetric domain with a total of $220 \times 1,470$ grid points was used with a stretched grid to cover a $1 \mathrm{~m} \times 1 \mathrm{~m}$ physical domain. The mesh spacing was distributed such that there was a uniform fine mesh with a size of $10 \mu \mathrm{m}$ covering the bubble/material area of interest where the interaction between bubble and material is important. The axisymmetric half-infinite computational domain was bounded by the material surface and far field grids away from the bubble. Transmission non-reflective boundary conditions, i.e. the flow variables are extrapolated along the characteristic wave direction, were imposed at the far field boundaries. On the axis of symmetry all physical variables such as density, pressure, velocity, and energy have zero gradients.

For the material dynamics computations, axisymmetry was also used, and the material was supposed to be in a circular plate with a radius of $1 \mathrm{~m}$ and a thickness of $0.01 \mathrm{~m}$ with discretized rectangular brick elements. As shown in Figure 6, a stretched grid with 220 elements in the radial direction, $r$, and 446 elements in the axial direction, $z$, were used to discretize the plate. Near the center of the plate $(r=0)$ where the high pressure loading occurs, fine elements of $10 \mu \mathrm{m}$ size were used. The motion of the nodes at the plate bottom was restricted in all directions. On the axis of symmetry the nodes were allowed to move only in the vertical direction. 
Figure 5: Axisymmetric fluid computational domain used for the computation of the bubble dynamics: full domain (right), zoomed area at the bubble location (left). The purple region is the inside of the deformed bubble with a well-developed reentrant jet at the axis of symmetry.

(a)

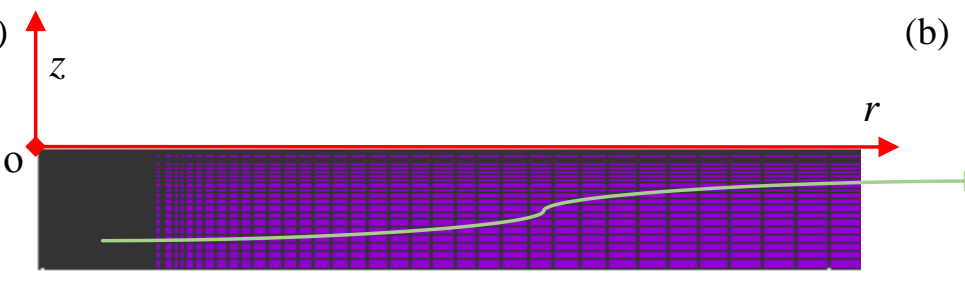

(b)

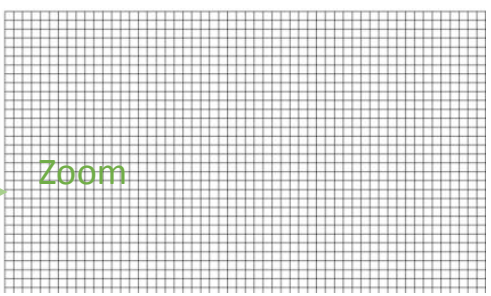

Figure 6. Finite element axisymmetric grid used in DYNA3D to study the material response to loads due to collapsing cavitation bubbles. (a) Coordinate system with stretched grid at far field, and (b) Zoom near (0,0) showing uniform mesh with 10 mm spacing.

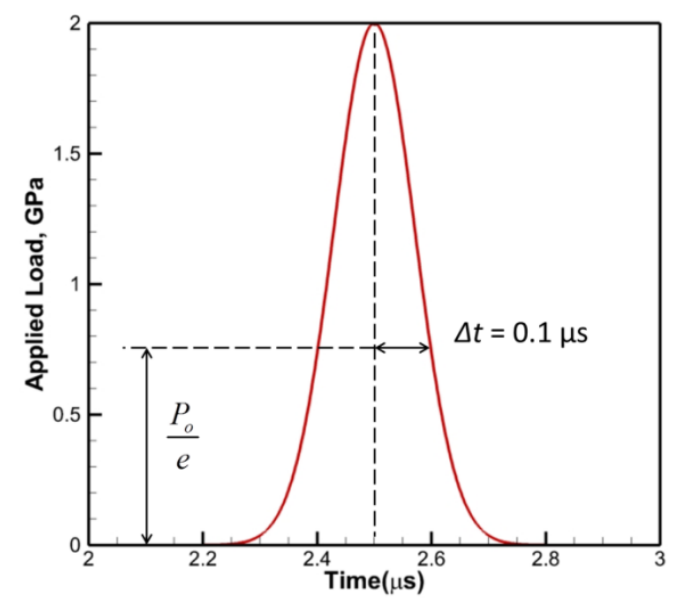

\section{Figure 7. Illustration of the Gaussian load varying in time. Peak pressure $2 \mathrm{GPa}, \Delta t=0.1 \mu \mathrm{s}$.}

\subsection{Synthetic loading}

Synthetic loading as described in Section 3.4 was also considered to investigate the resulting material response. Typical load ranges based on previous numerical and experimental studies of cavitation erosion [7, 8, 13, 26] were used. These were as follows: pressure load amplitudes between $1 \mathrm{MPa}$ and $2 \mathrm{GPa}$, load durations between $0.1 \mu \mathrm{s}$ and $2 \mu \mathrm{s}$, and load radial 

varying in time with peak at $t=2.5 \mu \mathrm{s}$ is shown in Figure 7 . Only one parameter of the synthetic load was varied at a time to produce one loading condition shown in the data below. Early results of this approach were presented in [33].

\section{Results and Discussion}

\subsection{FSI simulations of Pit Formation and Pit Geometry}

In order to examine the validity of inferring from the pit geometrical shape factor, $\xi$, the magnitude of the impulsive pressure generated by cavitation bubble collapse, we conducted an extensive series of CFD and material finite element (FEM) simulations of pit formation in a material exposed to two types of loads:

a) fully coupled fluid structure interaction (FSI) simulations of bubble dynamics and material response;

b) material deformation due to synthetic loading configurations where we varied systematically the amplitude, space extent, and duration of the load.

The FSI simulations briefly described above modelled bubble shape deformation, reentrant jet development, compressible impact of the jet on the material with generation of a water hammer impact and emission of a shock wave. Bubble size, standoff from wall, and collapse driving pressure functions were varied to cover a range of FSI conditions.

Figure 8, Figure 9 and Figure 10 illustrate some details of the bubble dynamics and interaction with the nearby material. This particular bubble dynamics and material conditions shown in the figures correspond to bubble of initial radius $R_{0}=50 \mu \mathrm{m}$, maximum radius $R_{\max }=2$ $\mathrm{mm}$, at a standoff distance of $\mathrm{d}_{0}=1.5 \mathrm{~mm}$ from the material, while the collapse driving pressure was $P_{d}=10 \mathrm{MPa}$. Figure 8 shows bubble shape outlines at different instants during the bubble collapse. These shapes were obtained from the boundary element method solution and clearly show the development of the reentrant jet and its fast motion towards the opposite side bubble before striking the material boundary. Jet speed, jet geometric characteristics and momentum all are provided by the solution as described in [7, 8, 9]. Figure 9 shows bubble shapes and pressure contours obtained with the compressible flow solution [39]. Very high pressures can be seen and are generated by liquid-liquid impact at the time of reentrant jet impact on the opposite side of the bubble $(\mathrm{t} \sim 0.05 \mu \mathrm{s})$ and at the time of the jet-material wall impact $(\mathrm{t} \sim 0.4 \mu \mathrm{s})$. Figure 10 illustrates the pressures generated at the center of the material liquid interface at the material origin of coordinates. The liquid-liquid impact event generates a localized high pressure region which expands quasi spherically to the wetted material surface (first pressure peak in Figure 10). The bubble ring left after the jet touchdown, shrinks then collapses generating another high pressure wave (second peak in Figure 10). Extensive details of this physics is described in [7, 8, 9]. The material responds by developing an indentation corresponding in time to the high pressure generation as illustrated in Figure 10. Due to the high pressure generated by the collapse, the material deforms elastically first, then exceeds the elastic limit resulting in unrecoverable plastic deformation and a pit, in this case of a depth of about $10 \mu \mathrm{m}$. The ensuing oscillations in the material deformation take a few tens of microseconds. 


\section{Figure 8. Bubble cross-section shape evolution in time during bubble collapse near the material wall (left), and} $3 D$ view of an instant prior to reentrant jet touchdown on the opposite side of the bubble (right).
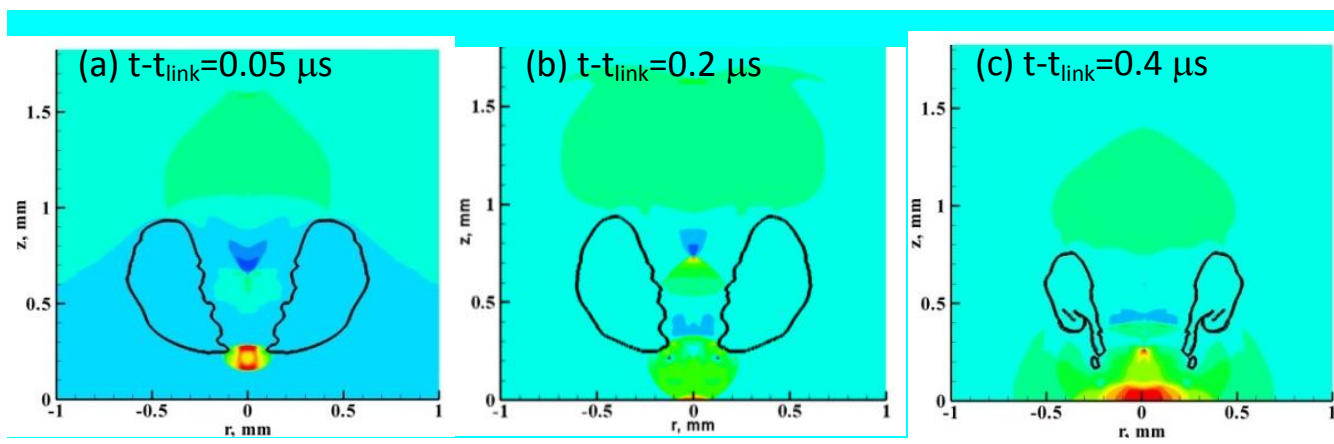

Pressure, MPa
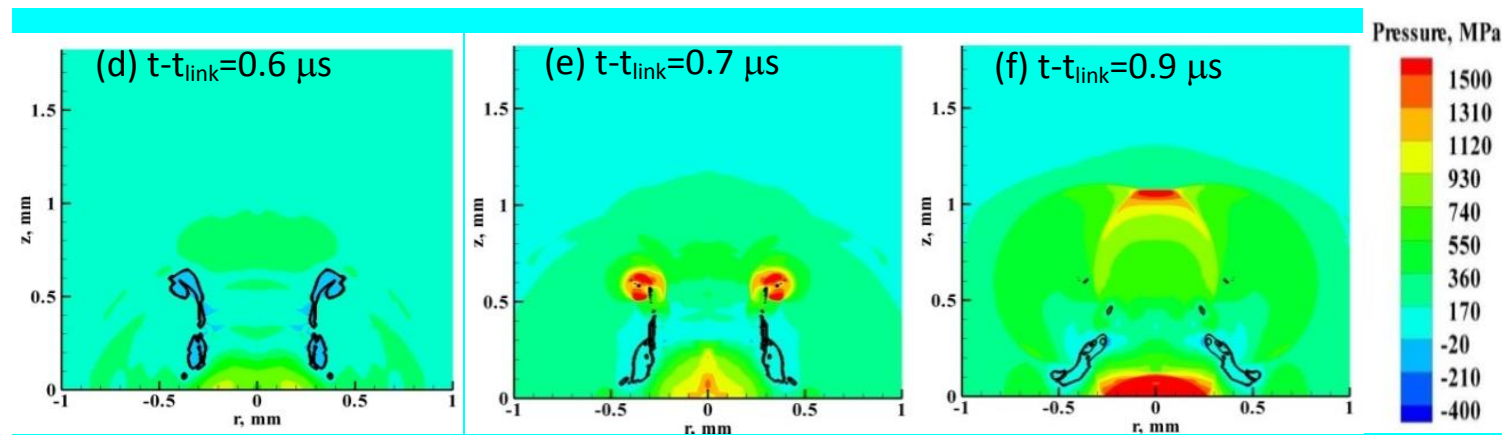

Figure 9. Example bubble shapes and pressure field during bubble collapse near a wall [8].

Initial bubble radius $R_{0}=50 \mu \mathrm{m}, R_{\max }=2 \mathrm{~mm}, d_{0}=1.5 \mathrm{~mm}$, the initial standoff $\bar{X}=0.75$, and collapse driving pressure $P_{d}=10 \mathrm{MPa}$ [8]. 

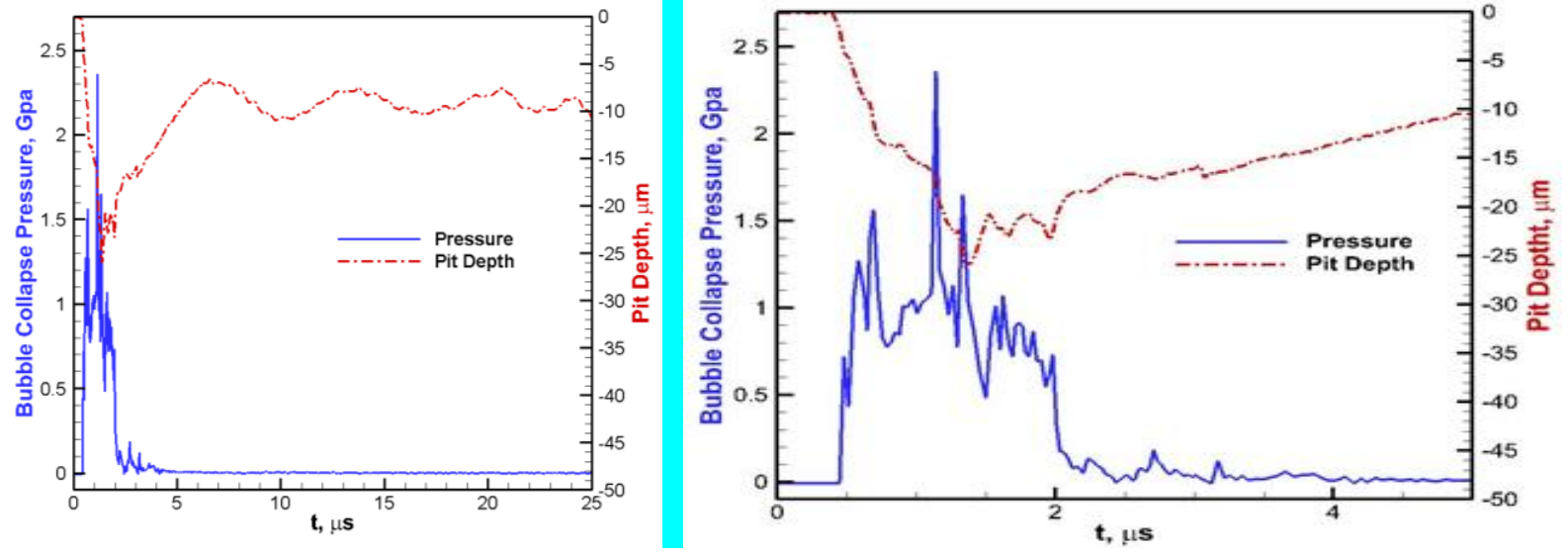

\section{Figure 10. Time history of pressure and vertical displacement of Al 7075 following the collapse of a cavitation} bubble [8] (left), and a zoom between $0 \mu$ s and $5 \mu$ s (right)

\section{a. Stress and Strain in the Material}

To understand the material response, stress and stain distributions inside the material are examined in Figure 11 and Figure 12. Here we consider an example of synthetic loading with duration $\Delta t=0.1 \mu \mathrm{s}$ and spatial radial extent, $\Delta r=100 \mu \mathrm{m}$. The amplitude of the selected impact is $P_{0}=2 G P a$ and the material is Stainless Steel A2205. Figure 11 (left) shows contours of the effective stress distribution, or von Mises stress, $\sigma_{v}=\sqrt{(3 / 2) s_{i j} s_{i j}}$, where $s_{i j}$ is the deviatoric stress tensor under the impacted surface when the maximum deformation of the material surface center is reached $(t=2.5 \mu \mathrm{s})$. The highest stresses are observed below the material surface at a depth of the same order as the load radial extent, $\Delta r$. This is a first important difference with the Tabor method assumptions.

Figure 11 (right) shows at the same time instant the distribution of the effective plastic strain defined as $\varepsilon_{v}=\int_{0}^{t} d \varepsilon_{v}, d \varepsilon_{v}=\sqrt{(2 / 3) d \varepsilon_{i j} d \varepsilon_{i j}}$, where $d \varepsilon_{i j}$ is the incremental plastic strain tensor. The effective strain also achieves a maximum at the same location below the surface.

As the load increases during the impulsive loading, the compressive stress below the surface may reach the material yield point (Figure 11). From there on, plastic deformations continues to increase until the maximum load is reached. If the load continues to rise beyond that point, an increasing volume of the material undergoes plastic deformation. Later on, as the load amplitude decreases, unloading occurs over a stress-strain line parallel to the initial elastic line, and a permanent indentation or "pit" remains once the load is fully relieved. Figure 12 shows the effective stress (left) and effective strain (right) at the end of the computation ( $t=10 \mu \mathrm{s}$ ) where the permanent deformation has been practically reached. The stress contours show a complex distribution of the residual stresses in the material due to the superposition of stress waves moving away from the center and other incoming waves, which have reflected from the bottom of the material thickness. The strains remaining in the permanently deformed material have a maximum located near the maximum residual stress location. By the time the permanent 

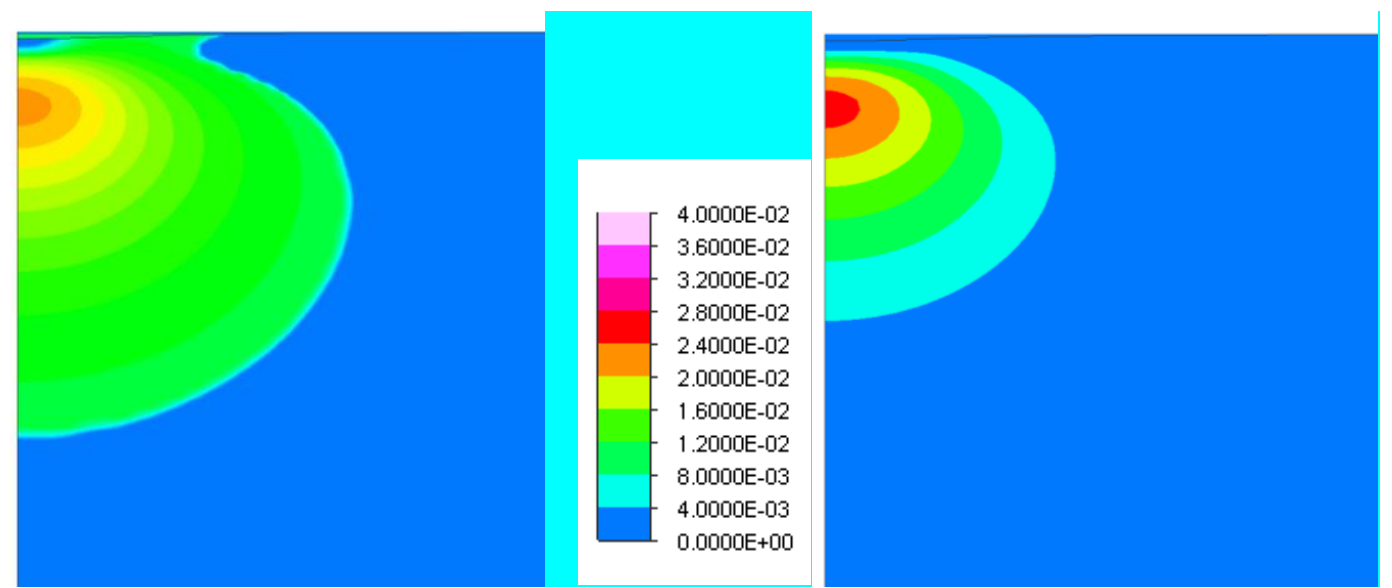

Figure 11. Effective stress (left) and effective plastic strain (right) at $2.5 \mu \mathrm{s}$. Synthetic loading function with duration $\Delta t=0.1 \mu \mathrm{s}$, spatial radial extent $\Delta r=100 \mu \mathrm{m}$, and amplitude $P_{0}=2$ GPa on Stainless Steel A2205.
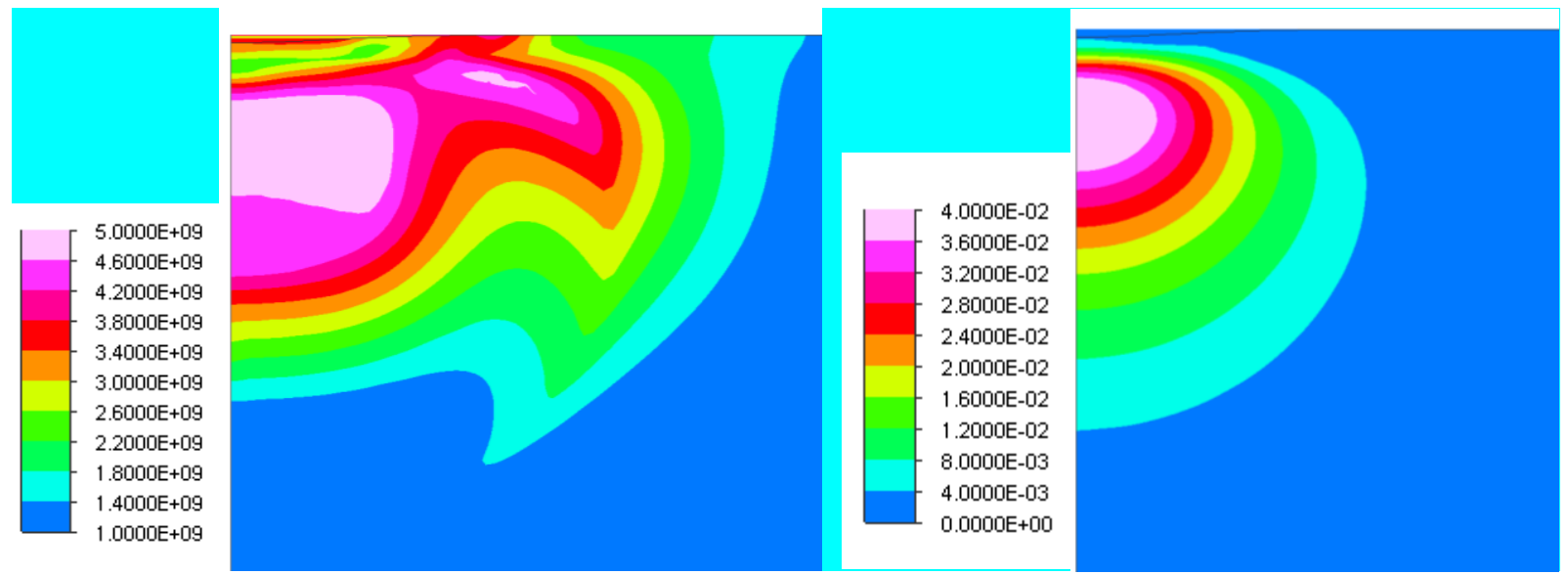

Figure 12. Effective stress (left) and effective plastic strain (right) at $10 \mu \mathrm{s}$. Synthetic loading function with duration $\Delta t=0.1 \mu$ s, spatial radial extent $\Delta r=100 \mu m$, and amplitude $P_{0}=2$ GPa on Stainless Steel A2205.

\section{b. Pit Depth and Diameter}

Examples of the permanent deformation predicted from FSI simulations are shown in Figure 13 through Figure 15 for both bubble collapse and synthetic loading cases. The profile of the permanent deformation generated on the surface resembles the experimentally observed pit shapes $[1,26]$ but are significantly different from the spherical shape assumed in the Tabor approach. The three figures also show the Tabor circle corresponding to the spherical shape assumed with the spherical indentation assumptions. In the case of a bubble generated pit, the shape is closer to a cone than a spherical sector. In addition, the pit shape varies significantly, for 
the same pressure driving bubble collapse conditions, with the standoff distance between the bubble and the wall as illustrated in Figure 15.

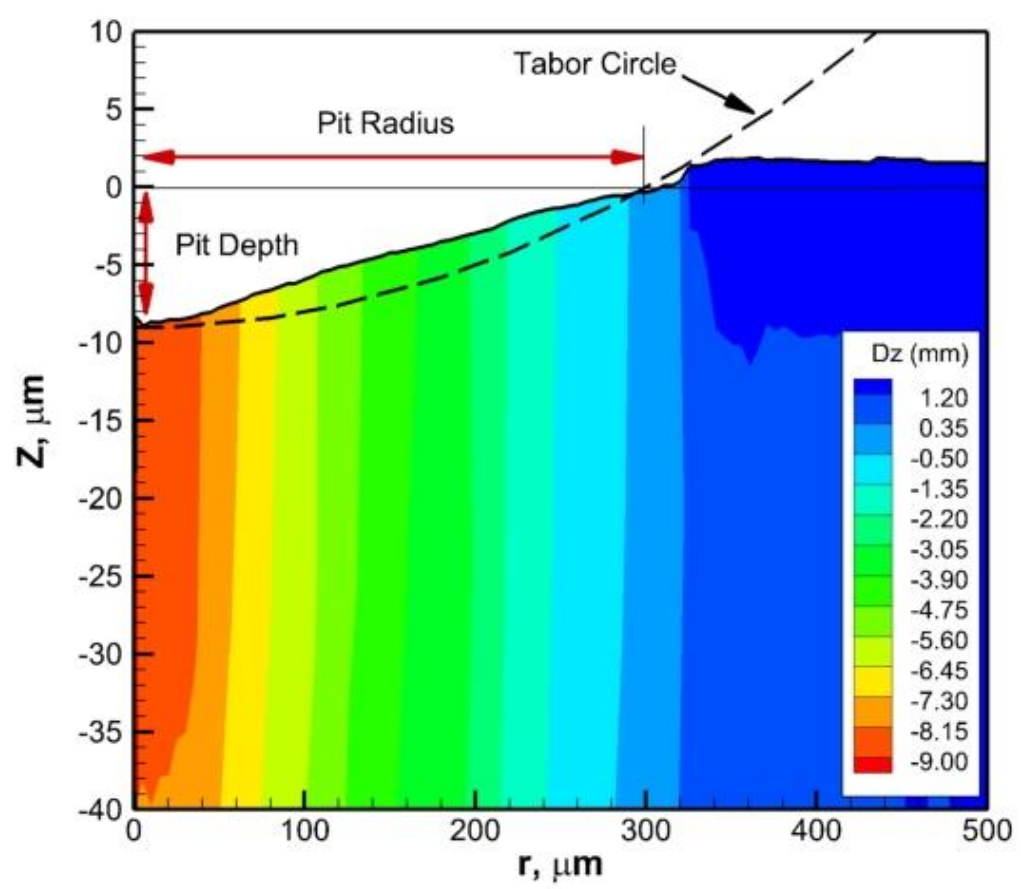

Figure 13. Profile of the permanent deformation an Al 7075 plate surface following the collapse of a cavitation bubble. $R_{0}=50 \mu \mathrm{m}, R_{\max }=2.0 \mathrm{~mm}, P_{d}=10 \mathrm{MPa}$, and $\bar{X}=0.75$.

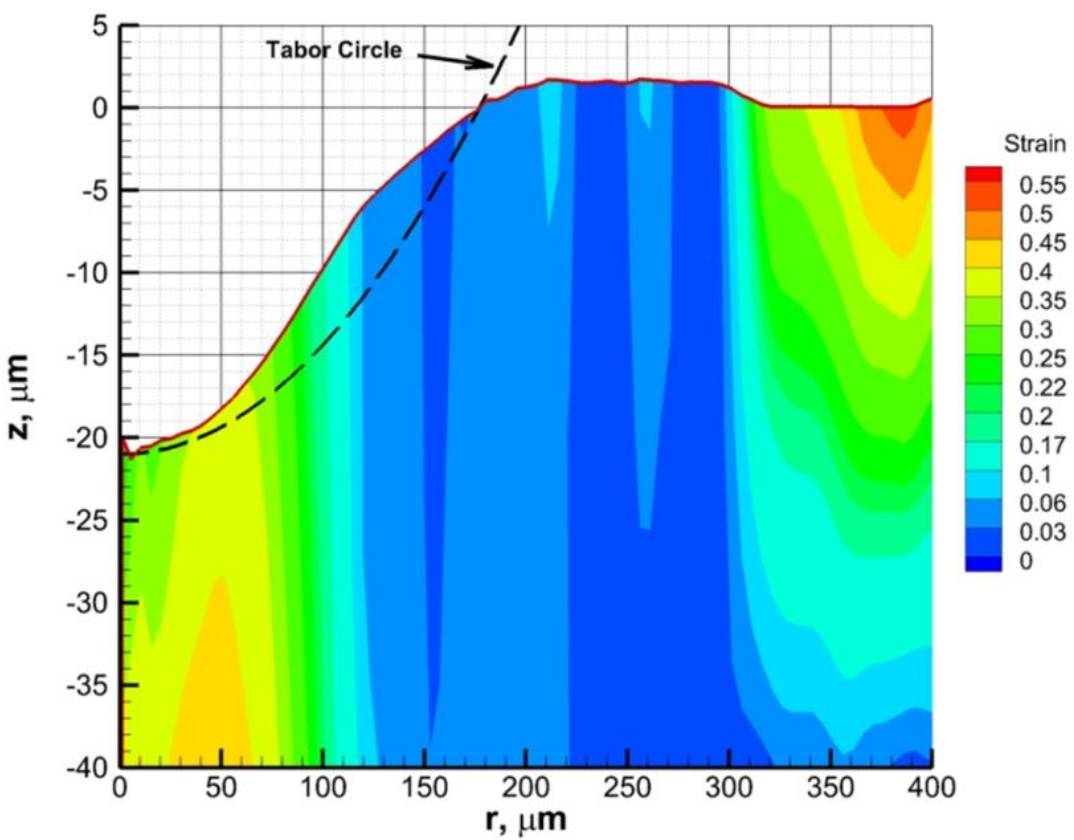

Figure 14. Predicted pit shape and circular shape used for Tabor formula. Synthetic loading $P_{0}=5$ GPa, $\triangle r=$ $100 \mu m, \Delta t=0.1 \mu$ s on Al 7075 . 


\subsection{Strain from Pit Shape by Tabor Formula}

Permanent pit shapes obtained from many simulations using both synthetic loading of various pressure peak magnitudes, durations, and radial extents and FSI simulations with various bubble sizes and standoff distances, were computed and analyzed. For all cases, the pit depth was determined from the maximum vertical displacement of the center point on the surface where the maximum deformation occurred. The determination of the pit diameter was based on a method similar to that conventionally used in pit experiments $[16,26]$, i.e. as the diameter of the region where the deformation depth exceeded a set depth criterion. For the numerical computations, a limit value of the deformation of $1 \mu \mathrm{m}$, which is of the same order as the height of the material grids near the material surface was selected. (Note that, obviously, any pits with a depth smaller than $1 \mu \mathrm{m}$ depth are screened out by such a criterion and actually by the numerical simulations itself when the grid size of the order or $1 \mu \mathrm{m}$ ). The cutoff criterion was used to avoid influence of tiny ripples on the surface from numerical simulations oscillations (similarly, from preexisting surface roughness in the case of experiments). The effect of this criterion on the results was measured and is actually minimal as seen in Table 2. Using the pit geometry in Figure 14, sensitivity to the pit diameter criterion was investigated by varying the cutoff value from $0.5 \mu \mathrm{m}$ to $3 \mu \mathrm{m}$. The resulting maximum strains obtained from the Tabor relations and the corresponding stresses are summarized in Table 2. It is seen that the used cutoff value for the determination of the pit diameter affects the strains between $-3.9 \%$ and $13.8 \%$. However, the predicted stress value is not affected too much, and the deviations vary only between $-0.7 \%$ and $2.3 \%$. As we will see below, these variations are orders of magnitudes less than the errors due to the method itself. 


\begin{tabular}{|c|c|c|c|c|c|c|c|}
\hline $\begin{array}{c}\text { Cutoff to } \\
\text { determine pit } \\
\text { diameter }(\mu \mathrm{m})\end{array}$ & $\begin{array}{c}D \\
(\mu \mathrm{m})\end{array}$ & $\begin{array}{c}\mathbf{h} \\
(\mu \mathrm{m})\end{array}$ & h/D & $\begin{array}{c}\varepsilon \\
\text { (Tabor) }\end{array}$ & $\begin{array}{l}\% \& \text { deviation } \\
\text { from } 1 \mu \mathrm{m} \text { case }\end{array}$ & $\begin{array}{c}\sigma \\
(\mathbf{M P a})\end{array}$ & $\begin{array}{l}\% \sigma \text { deviation } \\
\text { from } 1 \mu \mathrm{m} \text { case }\end{array}$ \\
\hline 0.5 & 346 & 21 & 0.0607 & 0.146 & $-3.9 \%$ & 596 & $-0.7 \%$ \\
\hline 1.0 & 332 & 21 & 0.0633 & 0.152 & $0 \%$ & 600 & $0 \%$ \\
\hline 2.0 & 312 & 21 & 0.0673 & 0.162 & $6.2 \%$ & 607 & $1.2 \%$ \\
\hline 3.0 & 292 & 21 & 0.0719 & 0.173 & $13.8 \%$ & 614 & $2.3 \%$ \\
\hline
\end{tabular}

Table 2. Sensitivity of the depth threshold in determining the pit diameter and the stress prediction by Tabor formula. The numerically obtained pit geometry in Figure 14 was used for this analysis.

Figure 16 gathers the results of all simulations in one plot and shows pit depths as functions of the magnitude of the peak loads applied to the material. The data covers a large range of loads and pits with the pit depth varying between a few microns and $50 \mu \mathrm{m}$. There is obviously a general trend of deeper pits as the peak load increases. However, there is wide scattering in the data with the variation in the loading conditions (i.e. different cavitation bubble history, size of the bubble, duration of the load, etc.). We should also highlight that, as expected, peak loads smaller than a critical load, which is material dependent, do not produce any pits. For such relatively low magnitude loads, the material deforms elastically and then recovers to its original shape once the load is removed, thus leaving no permanent deformation and no formation of a pit. This critical load level depends on the material, and it is of the order of the yield strength of the material.

Figure 17 shows the pit diameters versus the pressure peak load magnitude for the same computations. Again, there is wide scatter as the pit diameter is not a function of only the magnitude of the load. For small loads which generate local stress lower than the yield stress of the materials, there are no pits. Such data points were excluded from this plot.

According to the Tabor's formula, (4), the ratio $\xi$ of pit depth to pit diameter should be related directly to the peak load. To examine this, $\xi$ is plotted in Figure 18 as a function of the pressure peak load. There is some improvement compared to the pit depth alone or the pit diameter alone correlations, but the scatter is still extremely large placing in strong doubt for cavitation loading the use of $\xi$ as an index to determine the impact load magnitude. 
Figure 16. Pit depth vs. peak pressure for the four materials considered in this study under a set of cavitation bubble dynamics and synthetic loading conditions.

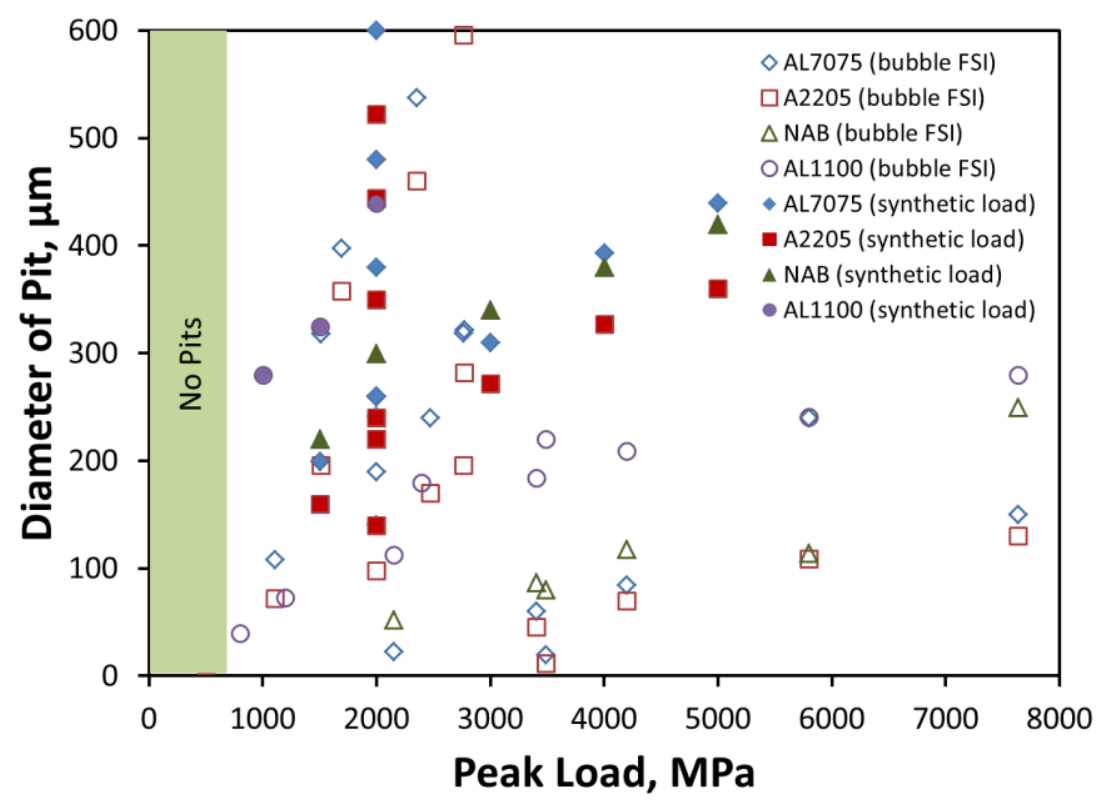

Figure 17. Pit diameter vs. peak pressure for the four materials considered in this study under a set of cavitation bubble dynamics and synthetic loading conditions.

Figure 18 collects as a function of the peak load imposed onto the materials the pit depth to diameter ratio, $\xi=h / D$, which according to Tabor's formula determines the load input. The figure clearly shows that the same impulsive pressure obtained from different bubble collapse 
conditions can produce very different values of $\xi$, which can vary by an order of magnitude (see for example the data points along the vertical dashed line in the figure). Similarly (see the red dashed horizontal line in the figure) loads which also varied by a factor of more than four produced the same value of $\xi$. Actually, for a cavitation event, for the same impulsive pressure peak level, the shape of the pressure function can vary significantly, both in space and time, for different bubble sizes, standoffs, and collapse driving pressure functions. In addition, for large strains, strain and stress distributions in the material under the pit deviate significantly from the simple relationship in Equation (4).

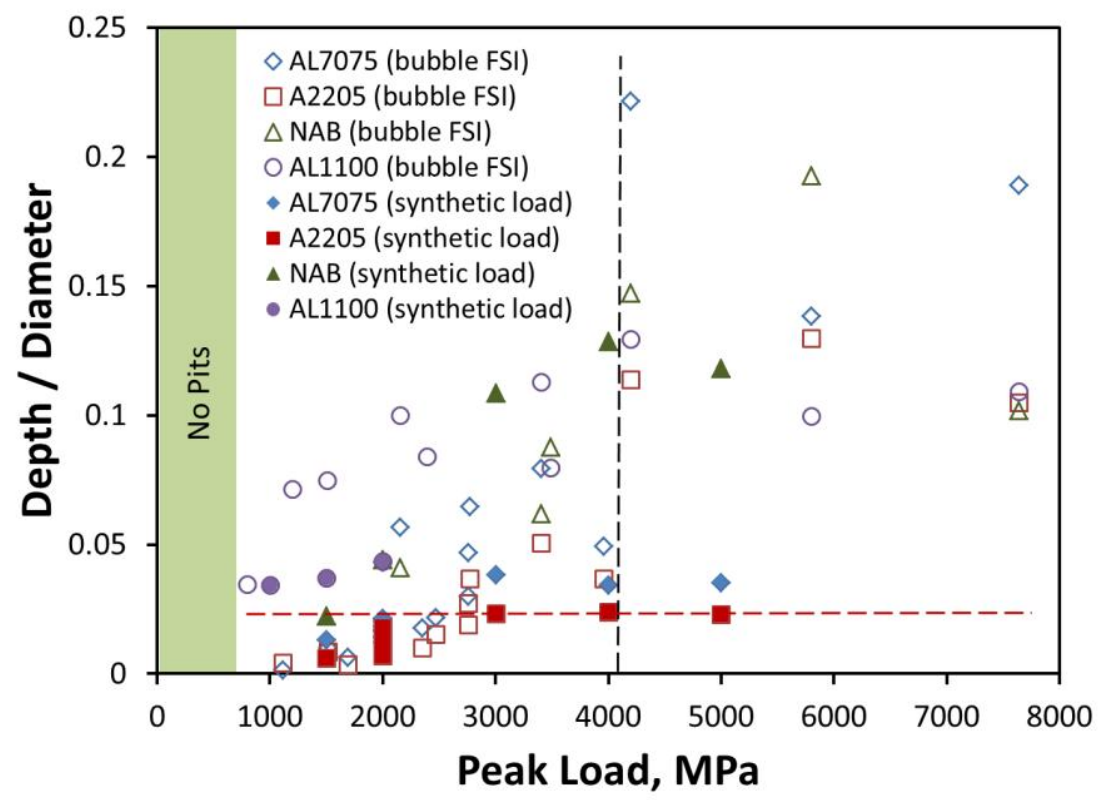

Figure 18. Pit depth to diameter ratio vs. peak pressure for all materials studied.

These significant deviations from a one-to-one relationship between the $h / D$ ratio and the imposed peak load are due to a combination of the following over-simplifying assumptions in the Tabor approach and in previous numerical work: spherical indentation, punctual or Gaussian pressure distribution, static load, and absence of interaction between the load and the material. Even for the simple Gaussian distribution, the radial extent of the load, $\Delta r$, and the load duration, $\Delta t$, have significant effects on the resulting deformation. We have investigated such effects in [33]. For instance, concerning the dynamic effect, the effect of the time duration of the synthetic load on the pits, while keeping all other variables the same, were presented in Figure 13 of [33] for Al 7075 and SS A2205. Pit diameter was not affected by changing $\Delta t$, however the depth to diameter ratios, $h / D$, changed significantly with $\Delta t$. Figure 19 presents this variation as a percentage deviation from the $h / D$ value at $\Delta t=2.0 \mu \mathrm{s}$. As can be observed, the depth to diameter ratio depends on the duration of the loading and can be up to $45 \%$ higher than the slow loading case. 


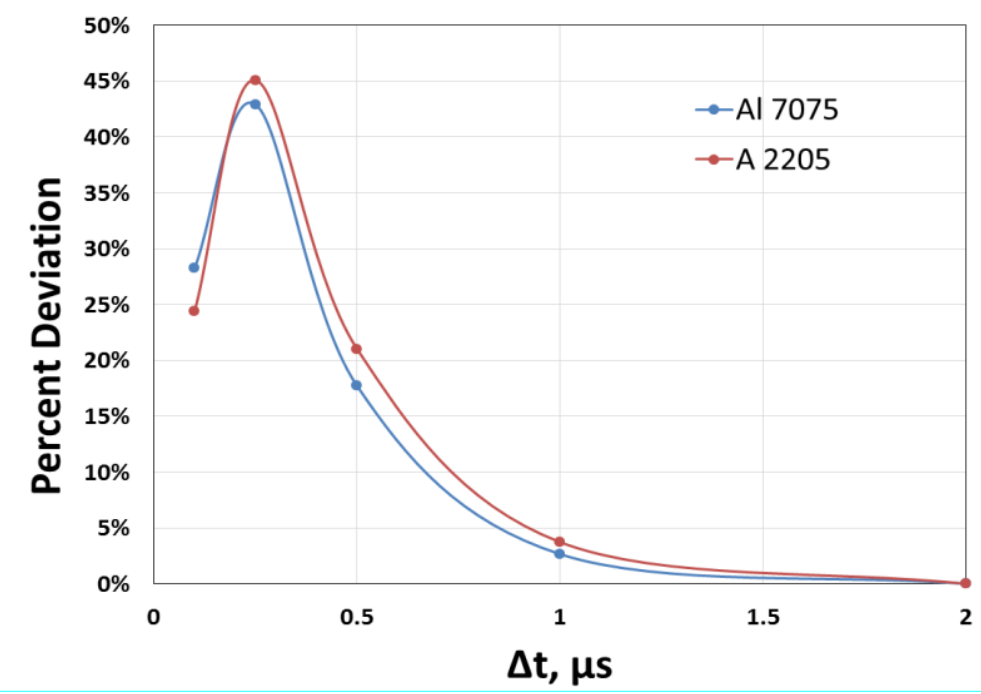

Figure 19. Percent deviation of pit depth to diameter ratio relative to the value at $\Delta t=2 \mu s \mathrm{vs}$. peak pressure duration for aluminum Al 7075 and stainless steel A2205.

This demonstrates that conclusions based on a single type of simplified synthetic loading ignoring time dependency and declaring one-to-one correspondence between load and deformation as in [31] are misleading and erroneous.

\subsection{Analysis of Maximum Stress from Tabor Relation}

To further analyze the above results and understand the reasons for the discrepancy, the mean strain around the pit was deduced from the Tabor relationship (4) and the ratio, $\xi$, between the pit depth and pit diameter, for all data shown in Figure 18. The mean strain thus obtained can be related to the maximum strain based on the assumption of Equation (5), i.e. exponential distribution of strain in the depth direction. The $\theta$ values reported in [46] were used here. These values are 2.0 for $\mathrm{Al}$ 7075, 2.8 for $\mathrm{NAB}$ and 2.2 for SS A2205. The value for Al 1100 is unknown, and we used the same value as for Al 7075 (2.0). One should therefore ignore the data for Al100 if this value may be too far from reality.

The $\xi=h / D$ ratios were converted to maximum strains using Equation (5) as shown in Figure 20. This provides maximum strains in the $10 \%$ to $20 \%$ range for peak loads up to $2 \mathrm{GPa}$. Larger strains exceeding 30\% were found for peak loads larger than $3 \mathrm{GPa}$. As the strain becomes larger, the distribution of the strains and stresses in the material around the pit deviate from a simple proportional relationship in the Tabor relationship and this contributes to the even larger scatter at higher loading. Figure 20a shows all data, including those where the strains exceeded the ultimate strains and where the computations are no longer valid. Figure 20b shows only valid data where conditions where strains exceeding the ultimate strains have been excluded.

Using the stress-strain curves in Figure 2, maximum stresses were deduced from the maximum strains and this is shown in Figure 21 as a function of the loading pressure peak magnitudes. This brings the data closer to a common curve by accounting for differences in the materials properties. However, wide scatter remains for well-defined pits and there is inability to differentiate small loads (below about $1 \mathrm{GPa}$ ), which did not produce well defined pits. The 
figure also shows that the values of the maximum stresses predicted from the pit geometries deviate very significantly from the corresponding applied pressure loads. While the peak loads varied from $1 \mathrm{GPa}$ to $8 \mathrm{GPa}$, the predicted maximum stresses were much lower varying between $500 \mathrm{MPa}$ and $1 \mathrm{GPa}$. This discrepancy comes from the difference between the applied maximum load at the surface and the maximum effective stress observed in the material which is more directly related to the amount of deformation and thus the pit depth. This is discussed further below.
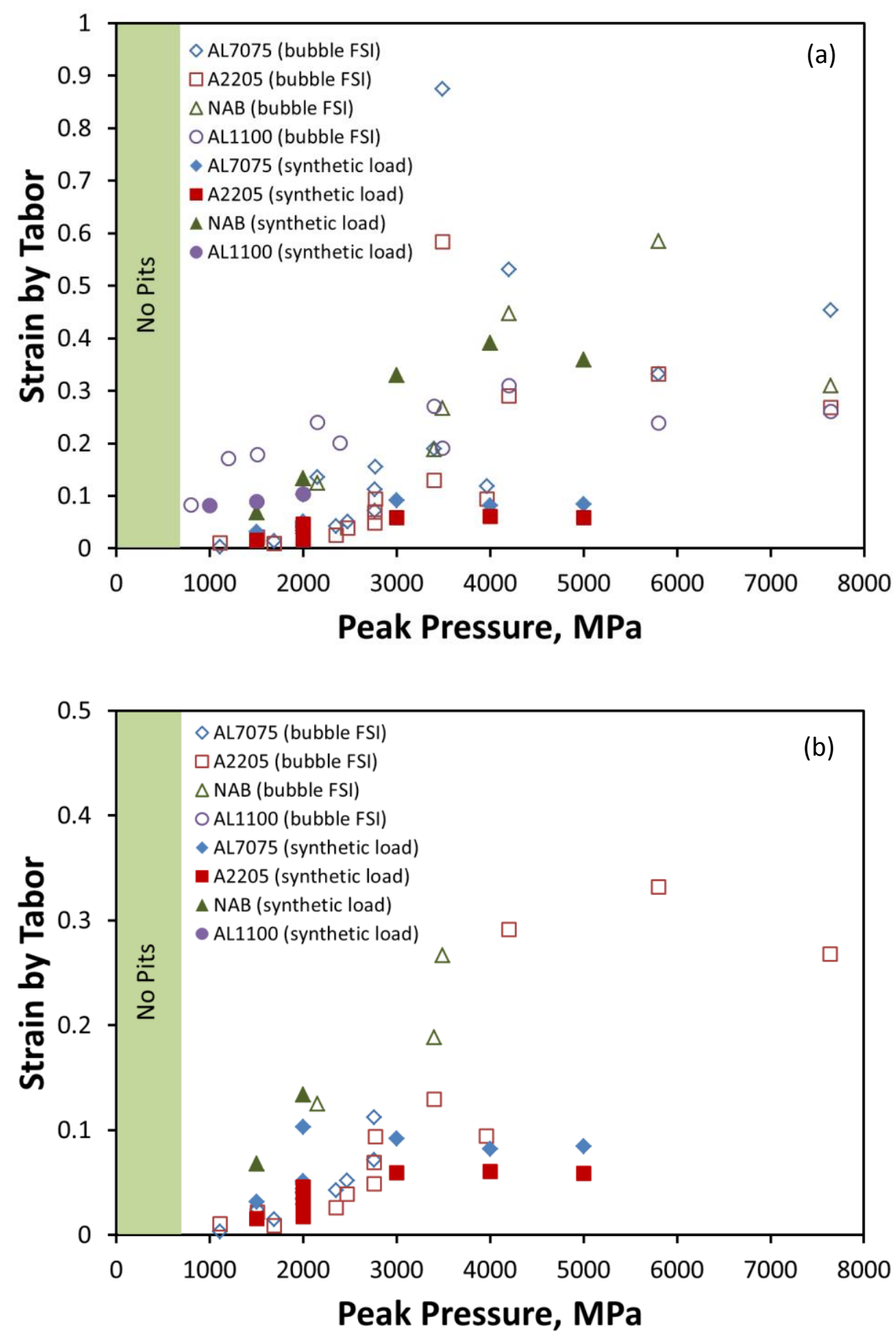
Figure 20. Maximum strains predicted from the Tabor formula using the computed pit depth to diameter ratios under the various loading condition of the four materials: a) all data points with some exceeding ultimate strain included, b) only data points below ultimate strain.

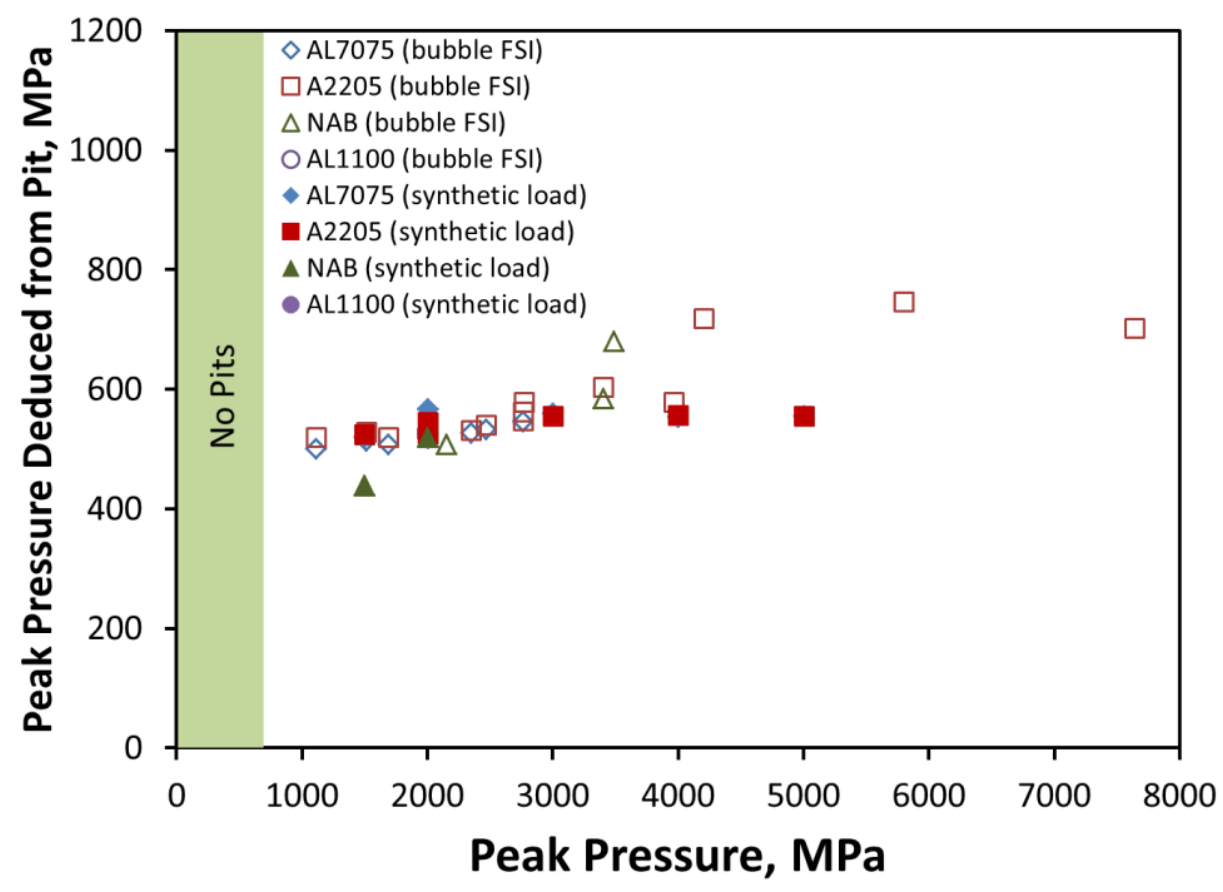

Figure 21. Stress predicted from Tabor formula using the computed pit depth to diameter ratios under the various loading condition of the four materials.

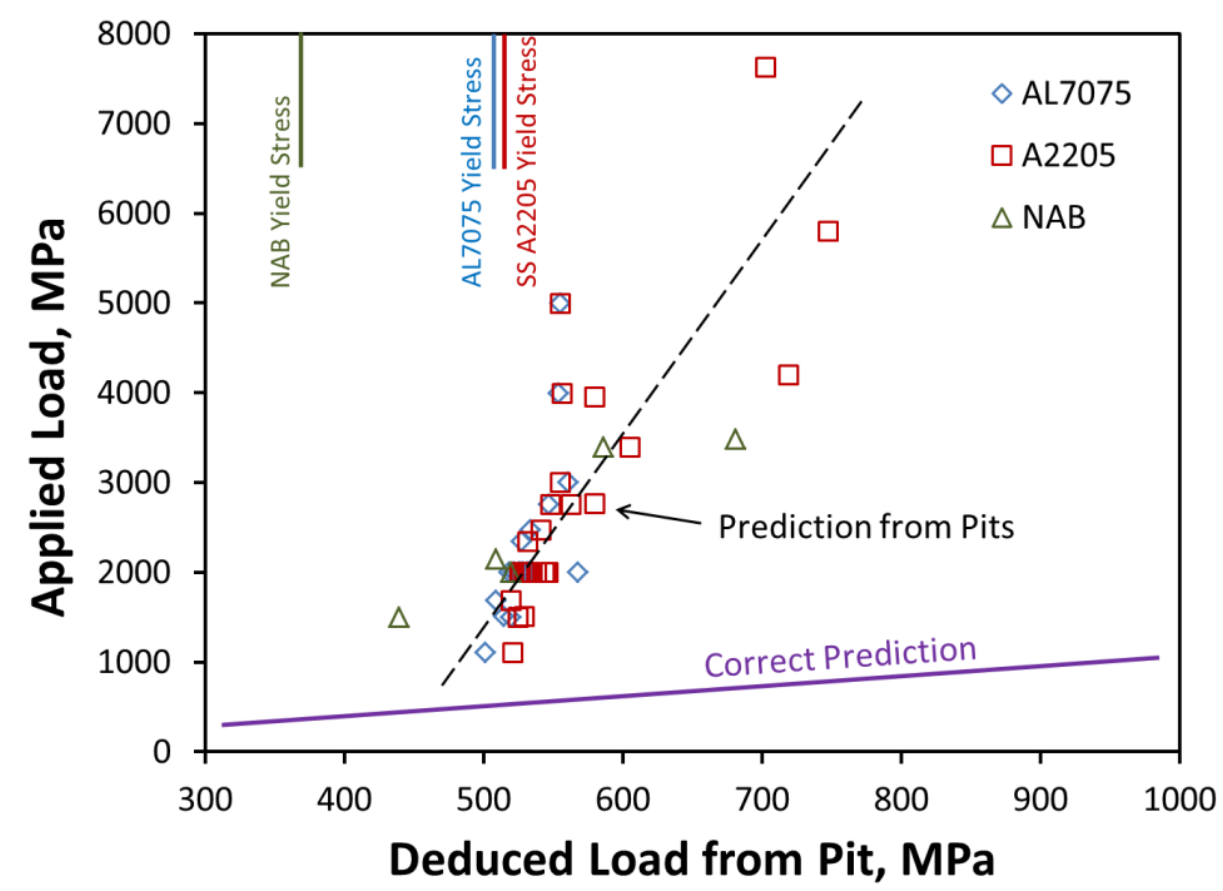

Figure 22. Comparison of the peak pressure applied to the material deduced from Tabor pit analysis with the actual applied pressure. 


\subsection{Comparison of Pressure Imposed, Pressure Deduced, and Maximum Effective Stress}

We discussed above the differences between the actual imposed pressure peaks and those deduced from the pit geometry and Tabor relations. Here we consider the maximum von-Mises (or maximum equivalent) stresses seen by the material. In this study, the maximum effective stress experienced by the material was extracted directly from the FEM simulations of the material dynamics for each of the simulations presented above. This maximum was obtained by scanning through each FEM solution both in time and in space. Figure 23 shows, for all four materials, the maximum effective stresses recorded in the material normalized by the yield stress of each material as functions of the applied pressure also normalized by the yield stress of each material. For clarity of the representation, only synthetic computations are shown but the results apply to all data. We can see that for small imposed loads, the maximum effective stress increases linearly with the peak load. However, once the maximum effective stress exceeds the yield stress of the material, the slope of the curve decreases due to the material experiencing permanent deformation in the plastic regime. With increased load, the slope in the plastic regime curves increases again. As expected with this representation, the linear part of the curve below the yield stress is common to different materials, but the plastic part above the yield stress is dependent on the material. 


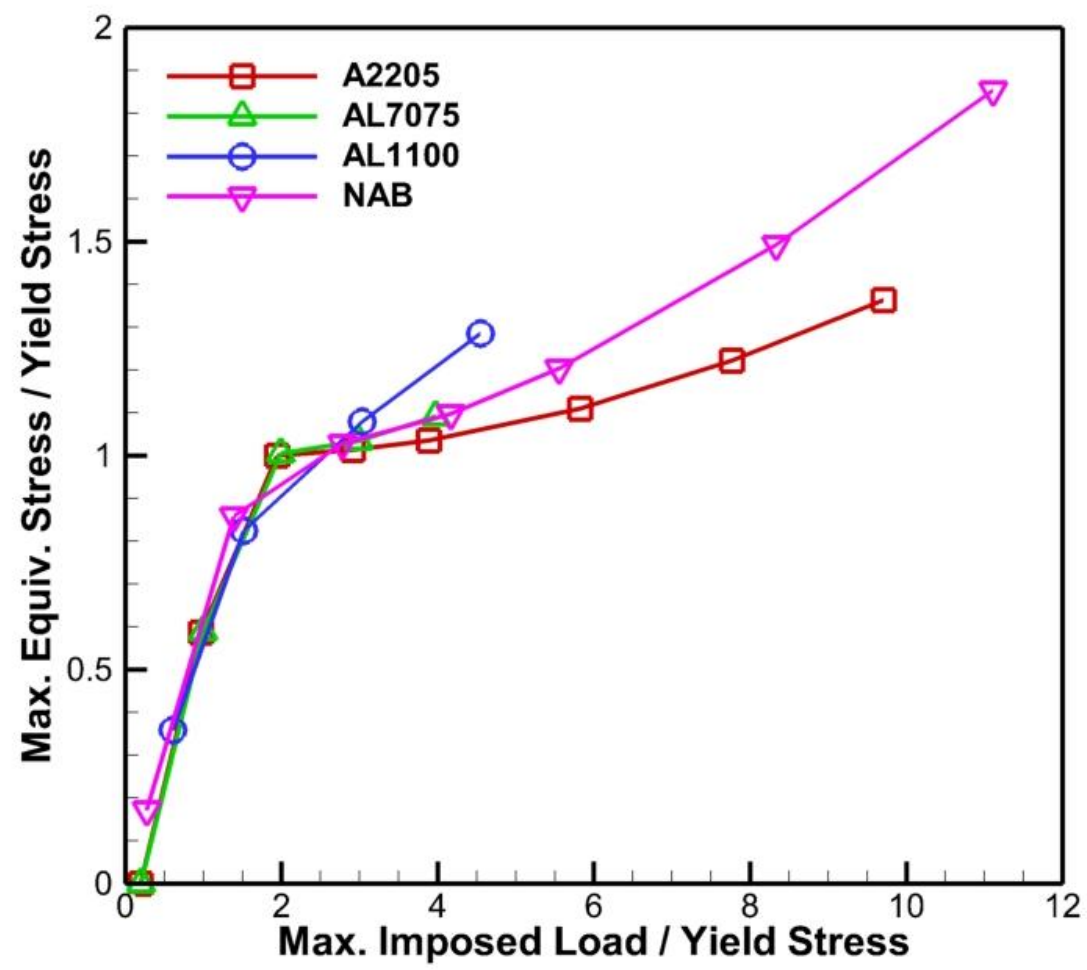

Figure 23. Maximum effective stress recorded in the material during the whole duration of simulation plotted against the maximum amplitude of the imposed load. Both the axes are normalized by the yield strength of respective materials. Synthetic loading with $\Delta t=0.1 \mu \mathrm{s}, \Delta r=100 \mu \mathrm{m}$.

The ratio of the pressures predicted from the pit geometrical shape ratio $\xi$ and the Tabor relation to the actually applied pressure peak is plotted in Figure 24. The data from the Tabor relation forms, for all materials, roughly a curved band significantly lower than 1 . The actual pressure divided by the pit-Tabor predicted pressure significantly increases with increasing loads exceeding 10 at the highest load values. Figure 24 also shows the numerically computed maximum effective stress inside the material for comparison. It is evident that the stress deduced from the Tabor relation agrees well with this maximum effective stress (von Mises stress) recorded in the material (not at the surface) and not the imposed load! The maximum effective stress is only $10 \%$ to $60 \%$ of the peak load depending on the magnitude of the load, which explains the above described discrepancies. Figure 25 further shows the level of accuracy of the Tabor-pit prediction of the maximum von-Mises stresses inside the material (not at the surface). The ratio is not actually one but it is much closer to one (by an order of magnitude - compare with Figure 24) than the ratio of predicted load to applied load. 


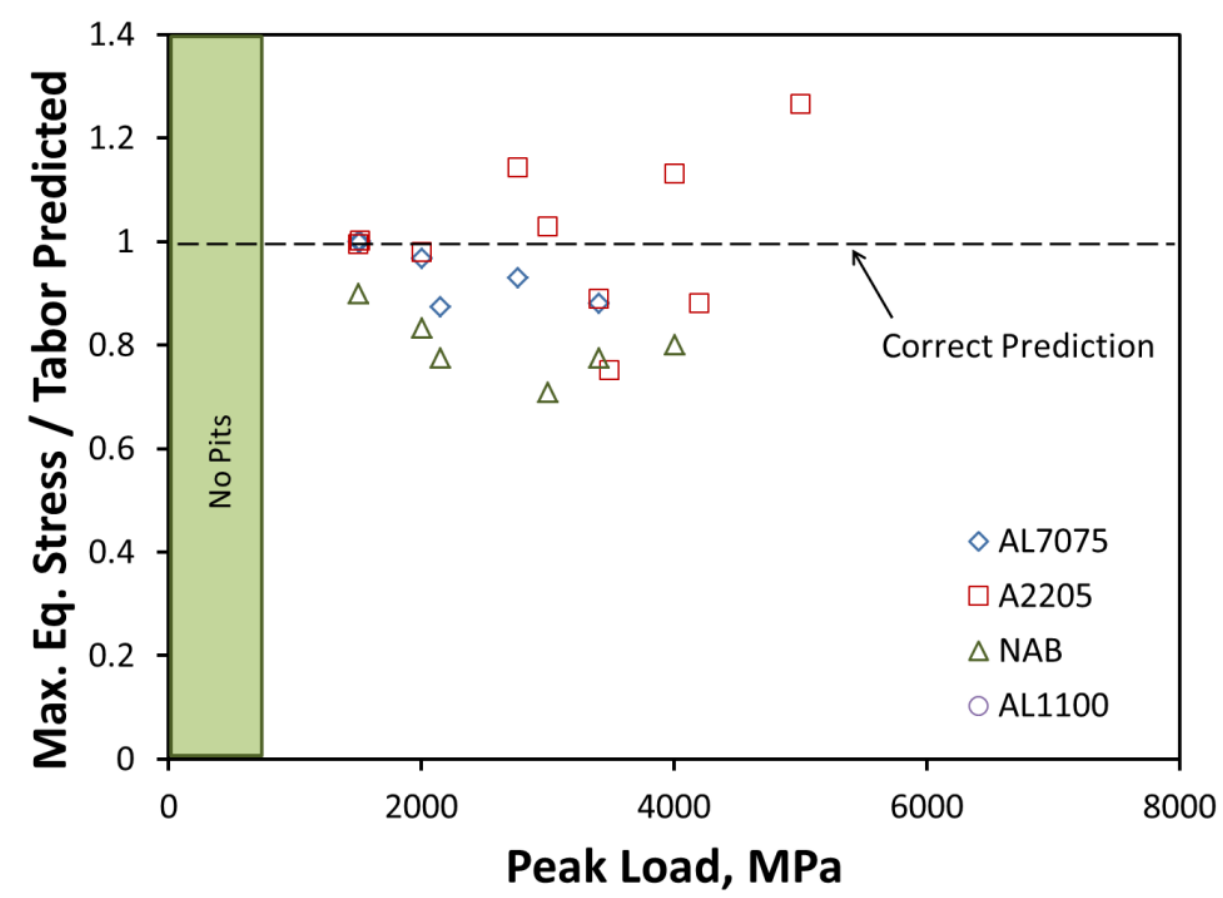

Figure 25. Ratio of maximum effective stress to Tabor-pit geometry deduced stress versus peak load.

Figure 24. Ratio of predicted peak load to actually imposed peak load and comparison with the ratio of maximum

effective stress to imposed peak load versus peak load.

- AL7075 (max. effective stress)

- A2205 (max. effective stress)

$\triangle \mathrm{NAB}$ (max. effective stress)

- AL1100 (max. effective stress)

$$
0
$$

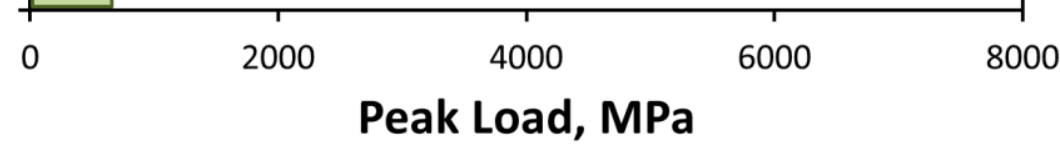




\section{References}

[1] K.-H. Kim, G. L. Chahine, J.-P. Franc, and A. Karimi, Advanced Experimental and Numerical Techniques for Cavitation Erosion Prediction, Series Fluid Mechanics and Its Applications, vol. 106. Springer, Berlin, Germany, 2014.

[2] R. T. Knapp, J. W. Daily, and F. G. Hammitt., Cavitation. London: McGraw-Hill, 1970.

[3] F. G. Hammitt, Cavitation and multiphases flow phenomena. McGraw-Hill, 1980.

[4] C. E. E. Brennen, Cavitation and bubble dynamics, vol. 9, no. 1. New York: Oxford University Press, 1995.

[5] J. R. Blake, B. B. Taib, and G. Doherty, "Transient cavities near boundaries. Part I. rigid boundary," J. Fluid Mech., vol. 170, pp. 479-497, 1986.

[6] S. Zhang, J. Duncan, and G. L. Chahine, "The Final Stage of the Collapse of a Cavitation Bubble Near a Rigid Wall,” J. Fluid Mech., vol. 257, pp. 147-181, 1993.

[7] G. L. Chahine, "Modeling of Cavitation Dynamics and Interaction with Material," in Advanced Experimental and Numerical Techniques for Cavitation Erosion Prediction, A. Kim, K-H, Chahine, G.L., Franc, J-P., Karimi, Ed. Berlin: Springer, 2014, pp. 123-173.

[8] C.-T. Hsiao, A. Jayaprakash, A. Kapahi, J.-K. Choi, and G. L. Chahine, "Modelling of Material Pitting from Cavitation Bubble Collapse," J. Fluid Mech., vol. 755, pp. 142-175, Aug. 2014.

[9] G. L. Chahine and C.-T. Hsiao, "Modeling Cavitation Erosion via Bubble-Material Interaction," Interface Focus, vol. 5, no. 5, 2015. 
[10] P. A. Lush, "Impact of a liquid mass on a perfectly plastic solid," J. Fluid Mech., vol. 135, pp. 373-387, 1983.

[11] H. Soyama, D. O. Macodiyo, and S. Mall, "Compressive residual stress into titanium alloy using cavitation shotless peening method," Tribol. Lett., vol. 17, no. 3, pp. 501-504, 2004.

[12] G. L. Chahine, C.-T. Hsiao, A. Kapahi, and J.-K. Choi, "Coupling bubble and material dynamics to model cavitation peening and pitting," in ICFD-2015, 2015.

[13] S. Singh, J.-K. Choi, and G. L. Chahine, "Characterization of Cavitation Fields From Measured Pressure Signals of Cavitating Jets and Ultrasonic Horns," J. Fluids Eng., vol. 135, no. 9, pp. 9111-91302, 2013.

[14] A. Thiruvengadam, "Handbook of cavitation erosion. Hydronautics Technical Report 7301-1.," Hydronautics Tech. Rep., no. 7301-1, 1974.

[15] P. Eisenberg, H. S. Preiser, and A. Thiruvengadam, "On the mechanisms of cavitation damage and methods of protection,” Trans. Soc. Nav. Archit. Mar. Eng., vol. 73, pp. 241286, 1965.

[16] J.-P. Franc, G. L. Chahine, and A. Karimi, "Pitting and Incubation Period," in Advanced Experimental and Numerical Techniques for Cavitation Erosion Prediction, vol. 160, K.H. Kim, G. L. Chahine, J.-P. Franc, and A. Karimi, Eds. Springer, Berlin, 2014, pp. 3770.

[17] C. A. Parsons and S. S. Cook, "Investigation into causes of corrosion or erosion of propellers," J. Am. Soc. Nav. Eng., vol. 31, pp. 536-541, 1919.

[18] R. T. Knapp, "Recent investigations of the mechanics of cavitation and cavitation damage," Trans. Am. Soc. Mech. Eng., vol. 77, pp. 1045-1054, 1955.

[19] R. T. Knapp, "Accelerated field tests of cavitation intensity," Trans. Am. Soc. Mech. Eng., vol. 80, pp. 91-102, 1958.

[20] J. M. Dorey, E. Laperrousaz, P. Avellan, P. Dupont, R. Simoneau, and P. Bourdon, "Cavitation erosion prediction on Francis turbines - Part 3 methodologies of prediction," in Hydraulic machinery and cavitation: proc. the XVIII IAHR Symposium, 1996.

[21] N. Berchiche, J.-P. Franc, and J. M. Michel, “A cavitation erosion model for ductile materials," in 4th International Symposium on Cavitation, CAV2001, 2001.

[22] M. Farhat, P. Bourdon, P. Lavigne, and R. Simoneau, "The Hydrodynamic aggressiveness of cavitating flows in hydro turbines," ASME Fluids Eng. Div. Summer Meet. FEDSM'97, 1997.

[23] M. L. Billet, "The specialist committee on cavitation erosion on propellers and appendages on high powered/high speed ships. Final report and recommendations to the 24th ITTC," in 24th Int. Towing Tank Conference. Vol. II., 2005.

[24] D. R. Stinebring, J. W. Holl, and R. E. A. Arndt, "Two aspects of cavitation damage in the incubation zone: Scaling by energy considerations and leading edge damage," J. Fluids Eng., vol. 102, pp. 481-485, 1980.

[25] M. Dular, B. Stoffel, and B. Sirok, "Development of a cavitation erosion model," Wear, vol. 261, pp. 642-655, 2006.

[26] A. Jayaprakash, J.-K. Choi, G. L. Chahine, F. Martin, M. Donnelly, J.-P. Franc, and A. Karimi, "Scaling Study of Cavitation Pitting from Cavitating Jets and Ultrasonic Horns," 
[27] A. Karimi and J.-P. Franc, "Modeling of material response," in Advanced Experimental and Numerical Techniques for Cavitation Erosion Predicition, K.-H. Kim, G. . Chahine, J.-P. Franc, and A. Karimi, Eds. Springer, 2014, pp. 163-180.

[28] D. Carnelli, A. Karimi, and J.-P. Franc, "Evaluation of the hydrodynamic pressure of cavitation impacts from stress-strain analysis and geometry of individual pits," Wear, vol. 289, pp. 104-110, 2012.

[29] D. Tabor, The Hardness of Metals. Oxford, United Kingdom: Clarendon Press, 1951.

[30] F. Pöhl, S. Mottyll, R. Skoda, and S. Huth, "Evaluation of cavitation-induced pressure loads applied to material surfaces by finite-element-assisted pit analysis and numerical investigation of the elasto-plastic deformation of metallic materials," Wear, no. Jan, Jan. 2015.

[31] S. C. Roy, J.-P. Franc, C. Pellone, and M. Fivel, "Determination of cavitation load spectra - Part 1: Static finite element approach," Wear, Sep. 2015.

[32] S. C. Roy, J.-P. Franc, N. Ranc, and M. Fivel, "Determination of cavitation load spectra Part 2: Dynamic finite element approach," Wear, Sep. 2015.

[33] J.-K. Choi, A. Jayaprakash, A. Kapahi, C.-T. Hsiao, and G. L. Chahine, "Relationship between space and time characteristics of cavitation impact pressures and resulting pits in materials," J. Mater. Sci., vol. 49, no. 8, pp. 3034-3051, Jan. 2014.

[34] K. M. Kalumuck, R. Duraiswami, and G. L. Chahine, "Bubble Dynamics Fluid-Structure Interaction Simulation by Coupling Fluid BEM and Structural BEM Codes," J. Fluids Struct., vol. 9, no. 8, pp. 861-883, 1995.

[35] G. L. Chahine, R. Duraiswami, and K. M. Kalumuck, "Boundary Element Method for Calculating 2-D and 3-D Underwater Explosion Bubble Behavior in Free Water and Near Structures," Nav. Surf. Warf. Center, Weapons Res. Technol. Dep., no. Report NSWCDD/TR-93/46, 1996.

[36] A. Wardlaw and J. A. Luton, "The Gemini Euler solver for the coupled simulation of underwater explosions," NSWCIH - Tech. Rep., no. 2500, 2003.

[37] C.-T. Hsiao and G. L. Chahine, "Development of compressible-incompressible link to efficiently model bubble dynamics near floating body," in Advances in Boundary Element \& Meshless Techniques XIV, 2013.

[38] K. M. Kalumuck, G. L. Chahine, and C.-T. Hsiao, "Simulation of Surface Piercing Body Couples Response to Underwater Bubble Dynamics Utilizing 3DynaFS(c), a ThreeDimensional BEM Code," Comput. Mech., vol. 32, no. 4-6, pp. 319-326, 2003.

[39] A. Kapahi, C.-T. Hsiao, and G. L. Chahine, "A multi-material flow solver for high speed compressible flow applications," Comput. Fluids, p. DOI: 10.1016/j.compfluid.2015.03.016, 2015.

[40] G. L. Chahine, R. Annasami, and C.-T. Hsiao, "Scaling of the Re-Entering Jet Parameters of an Underwater Explosion Bubble Below a Floating Structure," in 74th Shock and Vibration Symposium, 2002.

[41] A. B. Wardlaw and J. A. Luton, "Fluid-structure interaction mechanisms for close-in explosions," Shock Vib., vol. 7, pp. 265-275, 2000. 
[42] J. I. Lin, "DYNA3D: A nonlinear, explicit, three-dimensional finite element code for solid and structural mechanics. User manual," Laurence Livermore Natl. Lab., no. UCRL-MA107254, 2005.

[43] A. Jayaprakash, G. L. Chahine, and C.-T. Hsiao, "Numerical and Experimental Study of the Interaction of a Spark-Generated Bubble and a Vertical Wall," ASME J. Fluids Eng., vol. 134, no. 3, pp. 31301-31312, 2012.

[44] G. R. Johnson and W. H. Cook, "A constitutive model and data for metals subjected to large strains, high strain rates and high temperatures," in Proceedings of the 7th International Syposium on Ballistics, 1983, pp. 541-547.

[45] G. L. Chahine and Y. Shen, "Bubble Dynamics and Cavitation Inception in Cavitation Susceptibility Meters,” ASME J. Fluids Eng., vol. 108, no. 4, pp. 444-453, 1986.

[46] G. L. Chahine, J.-P. Franc, and A. Karimi, "Part 1: Cavitation and Cavitation Erosion Computational and Experimental Approaches," in Advanced Experimental and Numerical Techniques for Cavitation Erosion Prediction, K.-H. Kim, G. L. Chahine, J.-P. Franc, and A. Karimi, Eds. Springer, Berlin, 2014. 